\title{
Phase Direct CP Violations and General Mixing Matrices
}

\author{
Ling-Lie Chau \\ Physics Department, University of California, Davis
}

\begin{abstract}
I formulate expressions for amplitudes suitable for quantifying both modulus and phase direct $\mathrm{CP}$ violations. They result in Möbius transformation (MT) relations, which provide encouraging information for the search of direct $\mathrm{CP}$ violations in general. I apply the formulation to calculate the measurements of phase direct CP violations and strong amplitudes in $B^{\mp} \rightarrow K^{\mp} \pi^{ \pm} \pi^{\mp}$ by the Belle collaboration. For the formulation, I show a versatile construction procedure for $N \times N$ CabibboKobayashi-Maskawa (CKM) matrices, Pontecorvo-Maki-Nakagawa-Sakata (PMNS) matrices, and general unitary matrices. It clarifies the $3 \times 3$ cases and is useful for the beyond.
\end{abstract}

PACS numbers: 11.30.-j, 12.15.Ff, 13.20.He, 13.30.Eg

Introduction - $\mathrm{CP}$ violation studies and observations have had a long interesting history [1-11]. CP violation in $B^{\mp}$ published in [1] was the first of its kind, direct and without particle-antiparticle oscillation. Further, in multiparticle decays, the total amplitudes, $A_{(t o t)}$ and $\bar{A}_{(t o t)}$, are coherent sums of amplitudes for various final resonances and backgrounds, $A_{(k)}$ and $\bar{A}_{(k)}$,

$$
A_{(t o t)}=\sum_{k=1}^{n} f_{k} A_{(k)} \text { and } \bar{A}_{(t o t)}=\sum_{k=1}^{n} f_{k} \bar{A}_{(k)} \text {, }
$$

where $f_{k}$ are functions of invariant masses of some final particles. So phases of amplitudes can be measured, [1,2].

Here I derive general formulations capable of fully describe the phenomena and apply them to results of [1].

Expression A - Amplitudes, being complex valued, can always be expressed as

$$
A=|A| e^{i \phi} \quad \text { and } \quad \bar{A}=|\bar{A}| e^{i \bar{\phi}} .
$$

Direct CP violations are usually quantified by $\Delta_{c p} \neq 0$,

$$
\Delta_{c p} \equiv\left(|A|^{2}-|\bar{A}|^{2}\right) /\left(|A|^{2}+|\bar{A}|^{2}\right),
$$

where $A$ and $\bar{A}$ represent $A_{(t o t)}$ and $\bar{A}_{(t o t)}$ or $A_{(k)}$ and $\bar{A}_{(k)}$ in Eqs.(11). (The symbol $\Delta_{c p}$ is used, in stead of $\mathcal{A}_{c p}$, to avoid confusion with amplitudes.)

$\Delta_{c p}$ is insensitive to the phase of $\bar{A} / A$, which is convention dependent. To describe phase $\mathrm{CP}$ violation we should use amplitudes, denoted by $\bar{A}^{\prime}$ and $A^{\prime}$, which have the phase convention such that CP invariant amplitudes satisfy $\bar{A}_{i n v}^{\prime} / A_{i n v}^{\prime}=1 e^{i 0}$. Then

$$
\bar{A}^{\prime} / A^{\prime}=R_{c p} e^{i \Phi_{c p}} \equiv Z_{c p}, \quad-\pi<\Phi_{c p} \leq \pi,
$$

and their deviations from $Z_{c p, i n v}=1 e^{i 0}$ give full quantifications of direct $\mathrm{CP}$ violations, modulus and phase.

Expression B - Belle [1] used another modelindependent expressions for $B^{-}$and $B^{+}$respectively,

$$
\begin{aligned}
A^{\prime} & =a e^{i \delta_{B}}\left(1-b e^{i \varphi}\right), \quad \bar{A}^{\prime}=a e^{i \delta_{B}}\left(1+b e^{i \varphi}\right), \\
\Delta_{c p} & =-2 b \cos \varphi /\left(1+b^{2}\right) .
\end{aligned}
$$

I denote $B_{c p} \equiv b e^{i \varphi}$ and obtain MT conformal relations

$$
\begin{aligned}
\bar{A}^{\prime} / A^{\prime} & \equiv Z_{c p}=\left(1+B_{c p}\right) /\left(1-B_{c p}\right), \\
B_{c p} & =-\left(1-Z_{c p}\right) /\left(1+Z_{c p}\right) .
\end{aligned}
$$

Of the one-to-one and onto properties (circles/lines $\Leftrightarrow$ circles/lines) of MT, I point out some highlights. $b=0 \Leftrightarrow$ $Z_{c p}=1 e^{i 0}$. So $b \neq 0$ gives $\mathrm{CP}$ violation, in modulus and phase allocated by $b$ and $\varphi$. $\left[0<b<1, \varphi={ }_{0}^{\pi}\right] \Leftrightarrow \Phi_{c p}=$ 0 , thus all in $\Delta_{c p}= \pm 2 b /\left(1+b^{2}\right) ;[b \neq 0, \varphi= \pm \pi / 2]$ $\Leftrightarrow \Delta_{c p}=0\left(R_{c p}=1\right)$, thus all in $\Phi_{c p}= \pm 2 \arctan b$; maximal $\Phi_{c p}=\pi$ at $\left[1<b, \varphi={ }_{0}^{\pi}\right] ;$ maximal $\Delta_{c p}= \pm 1$ at $\left[b=1, \varphi={ }_{0}^{\pi}\right]$, where $\left[R_{c p}={ }_{\infty}^{0}, \Phi_{c p}\right.$ arbitrary $]$.

Belle [1] assumed the nonresonant parts to be $\mathrm{CP}$ invariant and measured all $b e^{i \varphi}$ and $\delta_{B}$. I calculate $Z_{c p}=R_{c p} e^{i \Phi_{c p}}$ (versus only $\Delta_{c p}=\mathcal{A}_{c p}$ calculated in [1]), thus revealing their measurements of direct $\mathrm{CP}$ violations both in the modulus and in the phase, shown at the end with other quantities I derive after giving the realization of Expression B in the KM framework [7].

Direct CP violation in the KM framework Direct $\mathrm{CP}$ violations come about naturally in the KM framework, as first established theoretically in $K$ mesons (the $s$ particles) [8]. Many other references and discussions can be found in the reviews of Particle Data Group (PDG) [12-14]. Here I give a self-contained discussion.

Weak decay amplitudes without particle-antiparticle oscillation are expressed as

$$
\begin{aligned}
& A=V_{u b} V_{u s}^{*} A_{1}+V_{t b} V_{t s}^{*} A_{2} \equiv z_{1} A_{1}+z_{2} A_{2}, \\
& \bar{A}=V_{u b}^{*} V_{u s} A_{1}+V_{t b}^{*} V_{t s} A_{2} \equiv z_{1}^{*} A_{1}+z_{2}^{*} A_{2},
\end{aligned}
$$

first for the $b \rightarrow s$ decays and then for decays with $z_{1}$ and $z_{2}$ as elements from the CKM matrix $\mathbb{V},[4,7]$.

One of the attributes of $\mathbb{V}$ is unitarity:

$$
\begin{aligned}
\sum_{m=1}^{3} V_{u_{m} d_{j}} V_{u_{m} d_{k}}^{*} & =\delta_{j k}, \quad j, k=1,2,3, \\
\sum_{j=1}^{3} V_{u_{m} d_{j}} V_{u_{n} d_{j}}^{*} & =\delta_{m n}, \quad m, n=1,2,3,
\end{aligned}
$$

where the letters $\left(u_{m}, d_{j}\right)$ denote the weak isospin doublets: $(u, d),(c, s),(t, b)$ quarks [or $\left(\nu_{e}, e\right),\left(\nu_{\mu}, \mu\right),\left(\nu_{\tau}, \tau\right)$ leptons involving the PMNS matrix, [15].]

Eqs.(910) can be derived by drawing quark diagrams and combing terms with the same $z$. Because of $\sum_{l=1}^{3} z_{l}=0$ conditions in Eqs.(1112), $A$ and $\bar{A}$ can always be expressed by two terms as in Eqs. (910) and in different ways. The strong amplitudes $A_{1}, A_{2}$ contain 
strong interactions to all orders. The relative phase of particle and antiparticle states is chosen such that the same strong amplitudes $A_{1}, A_{2}$ appear in $A, \bar{A}$. I will show that a suitable choice $\mathbb{V}^{\prime}$ can be made to obtain $A^{\prime}$, $\bar{A}^{\prime}$ (related to $A, \bar{A}$ by a phase transformation) so that phase direct $\mathrm{CP}$ violations can be quantified. That will be Expression $\mathrm{C}$.

In [16], I did a comprehensive study of direct CP violation for $c, b$, and $s$ particle decays. Writing

$$
\Delta_{c p}=\frac{-4 \operatorname{Im}\left(z_{1}^{*} z_{2}\right) \operatorname{Im}\left(A_{1}^{*} A_{2}\right)}{\left|z_{1} A_{1}+z_{2} A_{2}\right|^{2}+\left|z_{1}^{*} A_{1}+z_{2}^{*} A_{2}\right|^{2}},
$$

I found that all $\operatorname{Im}\left(z_{1}^{*} z_{2}\right)= \pm c_{1} c_{2} c_{3}\left(s_{1}\right)^{2} s_{2} s_{3} s_{\delta}$, in the notation of [7]. (This was four years before [17], whose parametrization has been called by PDG [12] the standard parametrization for the CKM and the PMNS matrices, thanks to the "advocation" and use by $[14,15]$.) The unique and ubiquitous $\left|\operatorname{Im}\left(z_{1}^{*} z_{2}\right)\right|$ found in $\Delta_{c p}$ were denoted by the symbol

$$
X_{c p} \equiv\left|\operatorname{Im}\left(z_{1}^{*} z_{2}\right)\right|=c_{12}\left(c_{13}\right)^{2} c_{23} s_{12} s_{13} s_{23} s_{\alpha_{13}},
$$

in $[17,18]$. I have been using it since. It touches upon aspects and developments of the theory complimentary to those the symbol $J$ does, [12-15]. It serves as a reminder that its relevance to experiments is through its role in direct $\mathrm{CP}$ violations.

Dividing the numerator and the denominator in Eq.(13) by $\left|z_{1}\right|\left|z_{2}\right|\left|A_{1}\right|\left|A_{2}\right|$ (assuming none of them are zero for now) and simplifying, we obtain

$$
\Delta_{c p}=-2 \sin \theta \sin \Theta /\left(l+l^{-1}+2 \cos \theta \cos \Theta\right),
$$

where

$$
\begin{aligned}
\sin \theta & \equiv \operatorname{Im}\left(z_{1}^{*} z_{2}\right) /\left|z_{1} z_{2}\right|, \quad r=\left|z_{2}\right| /\left|z_{1}\right|, \\
\sin \Theta & =\operatorname{Im}\left(A_{1}^{*} A_{2}\right) /\left|A_{1} A_{2}\right|, \quad R=\left|A_{2}\right| /\left|A_{1}\right| ; \text { or } \\
r e^{i \theta} & =z_{2} / z_{1} \equiv z_{21}, \quad R e^{i \Theta}=A_{2} / A_{1} \equiv A_{21} ;
\end{aligned}
$$

and $l \equiv r R$. The various $|\sin \theta|$ are

$$
\begin{aligned}
\sin \alpha_{d_{j} d_{k}} & =X_{c p} /\left|\left(V_{u d_{j}} V_{u d_{k}}^{*}\right)\left(V_{t d_{j}} V_{t d_{k}}^{*}\right)\right|, \\
\sin \beta_{d_{j} d_{k}} & =X_{c p} /\left|\left(V_{t d_{j}} V_{t d_{k}}^{*}\right)\left(V_{c d_{j}} V_{c d_{k}}^{*}\right)\right|, \\
\sin \gamma_{d_{j} d_{k}} & =X_{c p} /\left|\left(V_{c d_{j}} V_{c d_{k}}^{*}\right)\left(V_{u d_{j}} V_{u d_{k}}^{*}\right)\right|,
\end{aligned}
$$

and similarly for $\sin \alpha_{u_{m} u_{n}}, \sin \beta_{u_{m} u_{n}}$, and $\sin \gamma_{u_{m} u_{n}}$. (In the case of $d_{j} d_{k}$ being $b d$, the $\alpha, \beta, \gamma$ notations conform to those in [12].) Each set of $\alpha, \beta, \gamma$ with the subscripts $d_{j} d_{k}$ (or $u_{m} u_{n}$ ) is associated with the $d_{j} d_{k}\left(\right.$ or $u_{m} u_{n}$ ) orthogonal relation of Eqs. (11]12), thus the $d_{j} d_{k}\left(\right.$ or $\left.u_{m} u_{n}\right)$ triangle on the complex plane. To get the signs of various $\sin \theta$, it is best to use a specific parametrization, like the standard parametrization or its variations (which are needed for reasons to be discussed). Amplitudes of a particular set of decays, Eqs.(9[10), involve one particular triangle; yet, once $\mathrm{CP}$ violation is established in one decay (as has been) all $|z| \neq 0$ and all $\sin \theta \neq 0$.
Variations to the standard parametrization in the standard construction - To define $Z_{c p}$ and realize Expression B in the KM framework, I first show that the $z_{1}$ for a chosen $A_{1}$ can be made real and positive by using a suitable parametrization. [Note that all $A_{(k)}$ and $\bar{A}_{(k)}$ in Eq.(1) can be made to have the same $z_{1}$ ].

In [17], besides the standard parametrization of $\mathbb{V}, \mathrm{Ke}-$ ung and I found (by trials) a construction procedure for it: $\mathbb{V}=\mathbb{R}(23) \mathbb{U}(13) \mathbb{R}(12)$, one factor for each independent plane. $\mathbb{R}(j k)$ is the rotation matrix in the $j k$-plane and $\mathbb{U}(j k)$ is $\mathbb{R}(j k)$ with $\pm s_{j k} \rightarrow \pm s_{j k} e^{\mp i \alpha_{j k}}$,

$$
\mathbb{U}(13) \equiv\left(\begin{array}{ccc}
c_{13} & 0 & s_{13} e^{-i \alpha_{13}} \\
0 & 1 & 0 \\
-s_{13} e^{i \alpha_{13}} & 0 & c_{13}
\end{array}\right) .
$$

(Symbols $\alpha_{j k}$ are used, saving $\delta_{j k}$ for the Kronecker deltas.) So the standard parametrization is $R(23) U(13) R(12)$-parametrization. The procedure also provides variations: $\mathbb{V}^{\prime}=\mathbb{U}(23) \mathbb{R}(13) \mathbb{R}(12)$, or $\mathbb{V}^{\prime \prime}=\mathbb{R}(23) \mathbb{R}(13) \mathbb{U}(12)$, or $\mathbb{V} s$ with different ordering of (23) (13) (12). For $b \rightarrow s, b \rightarrow d$, and $s \rightarrow d$ decays, $\mathbb{V}^{\prime}$ gives real positive $z_{1}^{\prime}=V_{u b}^{\prime} V_{u s}^{\prime *}, V_{u b}^{\prime} V_{u d}^{\prime *}$, or $V_{u d}^{\prime} V_{u s}^{\prime *}$.

Here I digress to give a fuller explanation of the above and formulate (what I would "advocate" to call) the standard constructions for $N \times N$ CKM, PMNS, and general unitary matrices. Let us start with the following.

The Murnaghan construction of $N \times N$ general unitary matrices [19]:

$$
\mathbb{U}=\mathbb{F} \mathbb{A}, \quad \text { where } \quad \mathbb{A} \equiv \prod_{j<k \leq N} \mathbb{U}(j k),
$$

$\mathbb{F}=\operatorname{diag}\left(e^{i \phi_{1}}, e^{i \phi_{2}}, \cdots, e^{i \phi_{N}}\right)$ and the $\frac{1}{2} N(N-1)$ number (one for each plane in $N$ dimensions) of $\mathbb{U}(j k)$ are defined above Eq.(20). Different orderings of $\mathbb{U}(j k)$ give different (equally valid) parametrizations of $\mathbb{U}$.

$\mathbb{U}$ given by Eqs.(21) has all the attributes of a $N \times$ $N$ unitary matrix. For example, there are $\frac{1}{2} N(N-1)$ angles and $\left[\frac{1}{2} N(N-1)+N\right]=\frac{1}{2} N(N+1)$ phases.

Theorem 1: The core matrix $\mathbb{C}$, obtained from $\mathbb{A}$ by the maximal-phase-stripping similarity transformation (ST):

$$
\mathbb{C}=\mathbb{D} \mathbb{A D}^{\dagger}=\prod_{j<k \leq(N-1), m<N} \mathbb{U}^{\prime}(j k) \mathbb{R}(m N)
$$

with

$$
\mathbb{D}=\operatorname{diag}\left(e^{i \alpha_{1 N}}, e^{i \alpha_{2 N}}, \cdots, e^{i \alpha_{(N-1) N}}, 1\right),
$$

has the least possible number of phases under ST: $\left[\frac{1}{2} N(N-1)-(N-1)\right]=\frac{1}{2}(N-1)(N-2)$.

To prove the theorem, stick $\mathbb{D}^{\dagger} \mathbb{D}$ in-between all $\mathbb{U}(j k)$ of $\mathbb{A}$ in Eq.(21) and see that $\mathbb{D U}(j k) \mathbb{D}^{\dagger}=\mathbb{U}^{\prime}(j k)$ with $\alpha_{j k}^{\prime}=\alpha_{j k}-\alpha_{j N}+\alpha_{k N}$, giving $\alpha_{j N}^{\prime}=0$ and $\mathbb{U}^{\prime}(j N)=$ $\mathbb{R}(j N)$. So the ST of Eq. (22) maxes out the phase stripping from $\mathbb{A}$ [and all $\mathbb{A}$ with different ordering of $\mathbb{U}(j k)]$. 
Corollary 1: The phases in a core matrix $\mathbb{C}$ can be moved around by phase-moving ST (see an example of it later).

Using $\mathbb{C}$ we can make the following explicit constructions. Let us call them the standard constructions.

The standard construction of $N \times N$ general unitary matrices [revealing more phase structures than the Murnaghan construction, Eq.(21)]:

$$
\mathbb{U}=\mathbb{F D}^{\dagger} \mathbb{C D} \equiv \mathbb{F}^{\prime} \mathbb{C D}
$$

The standard construction of $N \times N$ CKM matrices for quarks:

$$
\mathbb{V}=\mathbb{C}^{q}
$$

Theorem 1 and Eq. (24) show by explicit construction how the usual phase counting works out. $(2 N-1)$ out of the $2 N$ phase freedoms of quark fields are used to strip away all that can be from the up-down quark mixing matrix $\mathbb{U}^{q}$ by phase transformations $(\mathrm{PT}): \mathbb{C}^{q}=\mathbb{F}^{\prime \dagger} \mathbb{U}^{q} \mathbb{D}^{\dagger}$, Eq.(24) to Eq.(25). So, always one phase is left free. It can be used to make one (only one) of the many $A_{1}$ in Eq.(11) real. In $\mathrm{K}$ decays, setting zero-isospin-change amplitude real is the $\mathrm{Wu}$-Yang phase convention [6].

What Keung and I found by trials in [17] is the $3 \times 3$ forerunner of this standard construction and [18] extended it to a $4 \times 4$ case. An example of Corollary 1 is the phase-moving $\mathrm{ST}, \mathbb{V}^{\prime}=\operatorname{diag}\left(1,1, e^{-i \alpha_{13}}\right) \mathbb{V} \operatorname{diag}\left(1,1, e^{i \alpha_{13}}\right)$ and $\alpha_{23}^{\prime}=-\alpha_{13}$. Other such relations among $\mathbb{V}, \mathbb{V}^{\prime}, \mathbb{V}^{\prime \prime}$ are left as exercises.

The standard construction of $N \times N$ PMNS matrices for Dirac leptons and for Majorana $\nu$ :

$$
\mathbb{V}^{l_{D}}=\mathbb{C}^{l_{D}}, \quad \text { and } \quad \mathbb{V}^{\nu_{M}}=\mathbb{C}^{\nu_{\mathbb{M}}} \mathbb{D} .
$$

Dirac lepton fields have the same phase freedoms as quarks fields, so $\mathbb{V}^{l} D$ is given by a core matrix as is $\mathbb{V}$ for quarks. However, Majorana neutrino fields do not have phase freedom [15], so only the phase freedoms of the Dirac leptons can be used to strip away $N$ phases from the Dirac-Majorana mixing matrix $\mathbb{U}^{\nu_{\mathbb{M}}}$ by one PT: $\mathbb{C}^{\nu_{\mathbb{M}}} \mathbb{D}=\mathbb{F}^{\prime \dagger} \mathbb{U}^{\nu_{\mathbb{M}}}$, Eq.(24) to Eq.(26). What has been adapted in neutrino research [15] is the $3 \times 3$ case of $[17]$ for $\mathbb{C}^{\nu_{\mathbb{M}}}$. All variations discussed here can also apply.

In Eq.(21) I can also use $\mathbb{U}=\mathbb{A}^{\prime} \mathbb{F}$ with $\mathbb{A}^{\prime} \equiv \mathbb{F} \mathbb{A} \mathbb{F}^{\dagger}$, follow similar procedure and obtain another core matrix $\mathbb{C}^{\prime}=\mathbb{D}^{\prime} \mathbb{A}^{\prime} \mathbb{D}^{\prime \dagger}$ for $\mathbb{U}$. I also have theorems that give different constructions with core matrices involving less than $\frac{1}{2} N(N-1)$ planes, like the Euler construction for $S O(3)$. However, I see no advantage over the standard construction for the uses discussed here. Further, I can use the core matrices to give spectral constructions for matrices. I give details of these results in [20].

Expression C - Representing CP invariant amplitudes by $A_{1} \neq 0$ and using the $\mathbb{V}^{\prime}$ in which $z_{1}^{\prime}=\left|z_{1}\right|$, I obtain Expression C:

$$
\begin{aligned}
& A^{\prime}=\left|z_{1}\right| A_{1}\left(1+z_{21} A_{21}\right)=d e^{i \delta_{1}}\left(1+l e^{i(\Theta+\theta)}\right), \\
& \bar{A}^{\prime}=\left|z_{1}\right| A_{1}\left(1+z_{21}^{*} A_{21}\right)=d e^{i \delta_{1}}\left(1+l e^{i(\Theta-\theta)}\right),
\end{aligned}
$$

where $d e^{i \delta_{1}} \equiv\left|z_{1}\right| A_{1}$ and relations given by Eqs.(16) still hold - good exercise to check; and

$$
\begin{aligned}
\bar{A}^{\prime} / A^{\prime} & \equiv Z_{c p}=\left(1+z_{21}^{*} A_{21}\right) /\left(1+z_{21} A_{21}\right), \\
A_{21} & =\left(1-Z_{c p}\right) /\left(z_{21}-z_{21}^{*} Z_{c p}\right) .
\end{aligned}
$$

Now the CP invariant $\bar{A}_{i n v}^{\prime} / A_{i n v}^{\prime}=1 e^{i 0}$. What we have done above is equivalent (and gives justification) to starting with $\mathbb{V}$ and making the phase change to amplitudes: $A^{\prime}=e^{-i \alpha_{1}} A$ and $\bar{A}^{\prime}=e^{i \alpha_{1}} \bar{A}$ with $e^{i \alpha_{1}} \equiv z_{1} /\left|z_{1}\right|$.

$Z_{c p}$ is in terms of the knowables (measurable in principle), $z_{21}$ and $A_{21}$, and is TM conformally related to $A_{21}$ (not $z_{21}$ ). Their one-to-one and onto mapping properties carry important information. $\operatorname{Im} z_{21}\left|A_{21}\right|=l \sin \theta=$ $0 \Leftrightarrow Z_{c p}=1 e^{i 0}$. So $l \sin \theta \neq 0$ assures $\mathrm{CP}$ violation, in modulus or phase allocated by $l \sin \theta$ and $\Theta$. $\Delta_{c p}$ is still given by Eq.(15). Since all $|z| \neq 0$ and all $\sin \theta \neq 0$ (i.e., $\operatorname{Im} z_{21}=r \sin \theta \neq 0$ and well defined), so in all decays direct $\mathrm{CP}$ violations happen everywhere on the whole $A_{21}$ complex plane except one point, $A_{21}=0$. [For example: at $\Theta=0, \Delta_{c p}=0$ and $\Phi_{c p}=-2 \arctan [l \sin \theta /(1+l \cos \theta)] ;$ and at $\Theta=\pi \pm \theta$ and $l=1, \Delta_{c p}= \pm 1$.] Further, for any value of $\operatorname{Im} z_{21}=r \sin \theta \neq 0, \mathrm{CP}$ violation can be large if $A_{21}$ cooperates. Eq.(30) gives the $A_{21}$ for a $Z_{c p}$ asked for.

Besides giving the conceptual understanding mentioned above and the realization of Expression $\mathrm{B}$ to be discussed below, Expression $\mathrm{C}$ also gives the possibility of finding $z_{21}, A_{21}$ and $\left|z_{1}\right| A_{1}$ from data via Eqs.(27/28); and provides versatile ways of analyzing data. [If the data are not sensitive to these many parameters, one can put in $z_{21}$ from [12] and find $A_{21}$ and $\left|z_{1}\right| A_{1}$.]

Realization of Expression B in the KM framework and derivation of amplitudes from Belle [1] - Using Eqs. 2728) of Expression C, decomposing $e^{ \pm i \theta}$ into real and imaginary parts, identifying

$$
\begin{aligned}
a e^{i \delta_{B}} & =\left|z_{1}\right| A_{1}\left(1+A_{21} r \cos \theta\right), \\
B_{c p} & =-i A_{21} r \sin \theta /\left(1+A_{21} r \cos \theta\right),
\end{aligned}
$$

I obtain the realization of Expression B, Eqs.(5), in terms of knowables in the KM framework.

Besides $\bar{A}^{\prime} / A^{\prime} \equiv Z_{c p}=R_{c p} e^{i \Phi_{c p}}$ mentioned earlier, Eqs.(7), I now can derive from Eqs.(31, 32)

$$
\begin{aligned}
A_{21} & =-B_{c p} /\left[i r \sin \theta+B_{c p} r \cos \theta\right] \quad \text { and } \\
A_{1} & =a e^{i \delta_{B}} /\left[\left|z_{1}\right|\left(1+A_{21} r \cos \theta\right)\right]
\end{aligned}
$$

in terms of $B_{c p}$. The MT conformal relations between $B_{c p}$ and $A_{21}$, Eqs.(32) 33), are anticipated from the MT relations that $Z_{c p}$ has both with $B_{c p}$, Eqs.(78), and with $A_{21}$, Eqs.(29)30. 
Substituting into Eqs. (3132) $\delta_{B}$ and $B_{c p} \equiv b e^{i \varphi}$ from [1], and $\sin \theta=-\sin \alpha_{b s} \approx-0.82, \cos \theta \approx-0.57$, and $r=\left|V_{t b} V_{t s}^{*}\right| /\left|V_{u b} V_{u s}^{*}\right| \approx 46$ (derived using $[12,14]$ and assuming all angles in the standard parametrization of $\mathbb{V}$ being in the first quadrant), I obtain the values of $A_{21} \equiv A_{2} / A_{1}$ and $\tilde{A}_{1} \equiv\left(\left|z_{1}\right| / a\right) A_{1}$, listed below together with $Z_{c p}=R_{c p} e^{i \Phi_{c p}}$ for four of the decays observed by [1] as examples: $B^{\mp}$ to $\{1\} K^{*}(892) \pi^{\mp},\{2\} K^{*}(1430) \pi^{\mp}$, $\{3\} \rho^{0}(770) K^{\mp},\{4\} f_{2}(1270) K^{\mp}$,

$$
\begin{aligned}
\{1\} Z_{c p} & =1.16 \exp (-i 0.048), \\
A_{21} & =0.0021 \exp (-i 1.8), \tilde{A}_{1}=0.98 \exp (-i 0.052) ; \\
\{2\} Z_{c p} & =0.93 \exp (-i 0.12), \\
A_{21} & =0.0019 \exp (i 2.5), \tilde{A}_{1}=0.96 \exp (i 0.99) ; \\
\{3\} Z_{c p} & =0.74 \exp (-i 0.46), \\
A_{21} & =0.0087 \exp (i 2.4), \tilde{A}_{1}=0.85 \exp (-i 0.24) ; \\
\{4\} Z_{c p} & =1.98 \exp (-i 0.34), \\
A_{21} & =0.011 \exp (-i 1.7), \quad \tilde{A}_{1}=0.93 \exp (i 2.2) .
\end{aligned}
$$

Only central values are shown. The proper way to find errors in $R_{c p}$ and $\Phi_{c p}$ is to analyze data distributions in $Z_{c p}$ by authors of Belle [1]. However, I did carry out various error calculations using statistical errors in $b$ and $\varphi$ given by [1] and noticed the following. When an error in $\varphi$ decreases (increases) modulus $\mathrm{CP}$ violation, it increases (decreases) phase CP violation; in contrast, when an error decreases (increases) $b$, both the modulus and the phase $\mathrm{CP}$ violations decrease (increase). The phase CP violation, $\Phi_{c p} \neq 0$, in case $\{2\}$ stood out, [21].

$A_{1}=\left(a /\left|z_{1}\right|\right) \tilde{A}_{1}$ can be derived once Belle publishes values of $a$, using partial rates and $f_{k}$ of Eq.(11). (Note the wide range of the central values of the moduli and phases of $\tilde{A}_{1}$ and $A_{21}$. The proper way to obtain them and their error analyses will be to fit data using Expression C, [21].) These $A_{1}$ and $A_{2}$ from experiments can be compared with theory. (For current theoretical calculation schemes, see $[22,23]$, e.g.). Alternatively, use $A_{1}$ and $A_{2}$ from theory in Eqs.(3132), then solve for $r$ and $\theta$, and compare them with those obtained elsewhere.

Conclusion - The formulations given here have general applications for studying phase and modulus direct $\mathrm{CP}$ violations and strong amplitudes in weak decays, beyond the results calculated here for $B^{\mp} \rightarrow K^{\mp} \pi^{ \pm} \pi^{\mp}$ of [1]. The Möbius (linear fractional conformal) transformation relations found here tell us that in the KM formulation, once a $\mathrm{CP}$ violation is established in one reaction (as has been), the amount of it (phase and modulus) in other decays is unrestricted by the CKM matrix, but solely dependent on how cooperative the strong amplitudes are. This new understanding is encouraging for the search of direct $\mathrm{CP}$ violations in general. The versatile procedure given here for the constructions of $N \times N$ CKM, PMNS, and general unitary matrices clarifies the $3 \times 3$ cases and is useful for the beyond.
[1] Belle collaboration: A. Garmash et al., Phys. Rev. Lett. 96, 251803 (2006), and references therein.

[2] BaBAr Collaboration: B. Aubert et al., Phys. Rev. D 72, 072003 (2005), and references therein.

[3] T.D. Lee, R. Oehme, and C.N. Yang, Phys. Rev. 106, 340 (1957).

[4] N. Cabibbo, Phys. Rev. Lett. 10, 531 (1963).

[5] J.H. Christenson, J.W. Cronin, V.L. Fitch, and R. Turlay, Phys. Rev. Lett. 13, 138 (1964).

[6] T.T. Wu and C.N. Yang, Phys. Rev. Lett. 13, 384 (1964).

[7] M. Kobayashi and T. Maskawa, Prog. Theor. Phys. 49, 652 (1973).

[8] J. Ellis, M.K. Gaillard, D.V. Nanopoulos, Nucl. Phys. B 109, 213 (1976); F.J. Gilman and M.B. Wise, Phys. Lett. B 83, 83 (1979); and citations thereof.

[9] NA31 Collaboration, H. Burkhardt et al., Phys. Lett. B 206, 169 (1988); NA48 Collaboration, V. Fanti et al., Phys. Lett. B 465, 335 (1999); KTeV Collaboration, A. Alavi-Harati et al., Phys. Rev. Lett. 83, 22 (1999).

[10] $\mathrm{B}_{\mathrm{A}} \mathrm{B}_{\mathrm{Ar}}$ Collaboration: B. Aubert et al., Phys. Rev. Lett. 93, 131801 (2005); Belle Collaboration: K. Abe et al., arXiv: hep-ex/0507045

[11] Belle Collaboration: C.C. Wang et al. Phys. Rev. Lett. 94, 121801 (2005). and $\mathrm{B}_{\mathrm{A}} \mathrm{B}_{\mathrm{AR}}$ Collaboration: B. Aubert et al., arXiv: hep-ex/0408099.

[12] Particle Data Group (URL: http://pdg.lbl.gov): K. Hagiwara, Phys. Rev. D 66, 010001 (2002); S. Eidelman et al., Phys. Lett. B 592, 1 (2004); W.-M. Yao et al., J. Phys. G 33, 1(2006); and references therein.

[13] H. Quinn and A. Sanda in [12] 2002; D. Kirkby and Y. Nir in [12] 2006; and references therein.

[14] F.J. Gilman, K. Kleinknecht, and B. Renk in [12] 2004; K. Kleinknecht and B. Renk, Phys. Lett. B 639, 612 (2006); and references therein.

[15] B. Kayser in [12] 2006; R.N. Mohapatra et al., arXiv:hep-ph/0510213 v2; and references therein.

[16] L.-L. Chau Wang, in Weak Interactions as Probes of Unification - 1980, eds. G.B. Collins, L.N. Chang, and J.R. Ficenec, AIP Conference Proceedings No. 72. p.419.

[17] L.-L. Chau and W.-Y. Keung, Phys. Rev. Lett. 53, 1802 (1984).

[18] F.J. Botella and L.-L. Chau, Phys. Lett. B 168, 97 (1986).

[19] F.D. Murnaghan, The Unitary and Rotation Groups, Spartan Books, 1962.

[20] L.-L. Chau, Basic Mathematics for the Physical Sciences; and L.-L. Chau, Group Theory for the Fundamental Laws of Physics; both to be published.

[21] I thank A. Garmash of the Belle collaboration [1] for thoughtful comments and discussions on this paper after its appearance on arXiv.org as hep-ph/0612101 and for their interest in future analyses of their data using $Z_{c p}$ and Expression $\mathrm{C}$ given in this paper.

[22] M. Beneke and M. Neubert, Nucl. Phys. B 675, 333 (2003); C.-W. Chiang, M. Gronau, Z. Luo, J.L. Rosner, and D.A. Suprun, Phys. Rev. D 69, 034001 (2004); references therein and citations thereof.

[23] Lattice QCD collaborations - Fermilab, HPQCD, MILC, and UKQCD: C.T.H. Davies et al., Phys. Rev. Lett. 92, 022001-1 (2004) and citations thereof. 